\title{
Efectividad de las TIC en el trabajo colaborativo para la metodología de clase inversa
}

Enviado: 10 de diciembre de 2020 / Aceptado: 24 de febrero de 2021 / Publicado: 12 de julio de 2021

MARCELA HERRERA MUESES

Ecuador

marce_lily@hotmail.com

\section{RESUMEN}

Se ha demostrado que el uso de las TIC, mediante la metodología de clase inversa, genera mayor efectividad y motivación en estudiantes, propiciando un incremento en el desarrollo de habilidades y destrezas que se ratifican en un Proceso de Aprendizaje Significativo (PAS). En esta ocasión se destaca el trabajo colaborativo como mecanismo esencial en el proceso de enseñanza-aprendizaje, tomando en consideración: trabajo en equipo, resolución de problemas, comunicación y desarrollo de proyectos, como factores primordiales que encajan y concatenan directamente con las demás fases de clase inversa; por tal razón se propone el uso de herramientas tecnológicas encaminadas al alcance de los estándares educativos planteados por el Ministerio de Educación del Ecuador enmarcados en un criterio pedagógico constructivista y sustentados en el diseño del aprendizaje del siglo XXI. Consecuentemente se plantea el objetivo, destacar la aplicación de actividades colaborativas en clase inversa junto con el uso de herramientas tecnológicas que apoyen al fortalecimiento de habilidades de trabajo en equipo en los estudiantes, incentivando su implementación en varios contextos educativos.

\section{ABSTRACT}

Effectiveness of ICT in Collaborative Work for Flipped Classroom Methodology

It has been shown that the use of ICT, through the flipped classroom methodology, generates greater effectiveness and motivation in students, leading to an increase in the development of skills and abilities that are ratified in a Significant Learning Process (SLP). On this occasion, collaborative work is highlighted as an essential mechanism in the teaching-learning process, taking into consideration: teamwork, problem solving, communication and project development, as primary factors that fit and link directly with the other phases of reverse class; for this reason, the use of technological tools aimed at achieving the educational standards set by the Ministry of Education of Ecuador framed in a constructivist pedagogical approach and based on the design of learning in the 21st century is proposed. Consequently, the objective is to highlight the application of collaborative activities in reverse class together with the use of technological tools that support the strengthening of teamwork skills in students, encou- 
Para verificar el logro de la aplicación de las TIC en la fase de trabajo colaborativo, se establece un análisis comparativo del rendimiento académico antes y después de usar mencionadas herramientas con base a la experiencia de los autores, utilizando una investigación aplicada, para lo cual se tomó como caso práctico la asignatura de Lengua y Literatura de tercero de Bachillerato General Unificado (BGU) en la Unidad Educativa Fiscal San Francisco de Quito, obteniendo resultados favorables en el rendimiento académico de los estudiantes y así logrando un PAS efectivo; en tal virtud, es factible promover la presente investigación en las diversas asignaturas de la institución y a la vez en otros centros educativos.

Palabras Clave: TIC, clase invertida, trabajo colaborativo, proceso aprendizaje significativo raging their implementation in various educational contexts. To verify the achievement of the application of ICT in the collaborative work phase, a comparative analysis of academic performance before and after using these tools is established based on the experience of the authors, using applied research, for which the subject of Language and Literature in the third year of the General Unified Baccalaureate (BGU) at the San Francisco de Quito Public Education Unit was taken as a practical case, obtaining favourable results in the academic performance of students and thus achieving an effective PAS; In such virtue, it is feasible to promote the present investigation in the diverse subjects of the institution and at the same time in other educational centres.

Keywords: TIC, flipped classroom, collaborative work, significant learning process

\section{INTRODUCCIÓN}

El mundo de hoy necesita un cambio sistemático en los procesos educativos, en los cuales los estudiantes desarrollen habilidades del siglo XXI y competencias digitales.

Según Calzadilla (2002), el uso de tecnología en educación permite a los estudiantes transmitir información inmediata, trabajar con documentos conjuntos que faciliten la toma de decisiones y la solución de problemas.

En concordancia con lo expuesto, se contextualiza al trabajo colaborativo dentro de las llamadas habilidades del siglo XXI. IBEC (2019), afirma que "la habilidad de trabajar de manera efectiva y creativa con los miembros del equipo y compañeros de clase, independientemente de las diferencias en cultura y estilo, es una habilidad esencial para la vida del siglo XXI" (p. 2).

Ferreiro (2012), menciona que "toda actividad humana por su esencia es social y por tanto grupal" (p. 14). La educación es el peldaño que moldea a la sociedad en su conjunto, por ende, es la base fundamental para forjar el sentimiento de cooperación entre estudiantes para 
un bien común que garantice una sociedad armónica y de paz. Tomando como base el eje transversal de la formación de una ciudadanía democrática, el Ministerio de Educación (2010) manifiesta:

El desarrollo de valores humanos universales, el cumplimiento de las obligaciones ciudadanas, la toma de conciencia de los derechos, el desarrollo de la identidad ecuatoriana y el respeto a los símbolos patrios, el aprendizaje de la convivencia dentro de una sociedad intercultural y plurinacional, la tolerancia hacia las ideas y costumbres de los demás y el respeto a las decisiones de la mayoría. (p. 17)

El trabajo colaborativo según Férez (2005), promueve el aprendizaje significativo, además desarrolla habilidades cognitivas en los estudiantes, quienes se involucran de mejor manera en la sociedad, posibilitando que las actividades colaborativas sean integradoras en un fin común.

No se puede desestimar el hecho de que nos desenvolvemos en la sociedad del conocimiento, por ende, la información es inmediata y va de la mano con el uso adecuado de TIC, por ello es necesario posicionar al trabajo colaborativo como referente del uso de herramientas digitales en el desarrollo de una clase.

A partir del año lectivo 2016-2017 en la Unidad Educativa Fiscal San Francisco de Quito, se aplica la metodología de clase inversa como respuesta a la necesidad de cambio de paradigmas educativos en favor de los estudiantes. El punto de partida ha sido justamente el desapego de patrones tradicionales impregnados tanto en docentes como estudiantes, quienes han respondido de manera favorable a tal cambio, logrando la apropiación del proceso de enseñanza-aprendizaje que prioriza al estudiante como gestor de su conocimiento con el indudable apoyo del docente.

Es así que se presentó el artículo denominado las TIC en el desarrollo de clase inversa: experiencia Unidad Educativa Fiscal San Francisco de Quito (publicado en el 2019 en la revista Conrado indexada con diversas revistas científicas como: Scielo, Doaj, Latindex entre otras), con el siguiente link https://conrado.ucf.edu.cu/index.php/conrado/article/view/1133/1140 siendo este el primer paso para la realización de un análisis crítico de su ejecución, a la vez adjudicada a una investigación aplicada, midiendo el grado de satisfacción de los involucrados y el mejoramiento del rendimiento académico de los estudiantes.

Los resultados obtenidos son favorables y tienden a mejorar, es así que la institución ha sido auditada por el Ministerio de Educación del Ecuador, organismo rector del ámbito educativo a nivel nacional, quienes, en octubre del 2019 verifican y evalúan el cumplimiento de 
los estándares educativos establecidos en el currículo, junto con estándares de aprendizaje de gestión escolar y desempeño profesional.

Para dicha auditoría se toma en consideración el "Manual para la implementación y evaluación de los estándares de calidad educativa", el cual destaca indicadores de calidad representados por un color y su significado. (Ver Figura 1).

La Unidad Educativa Fiscal San Francisco de Quito posteriormente a la auditoría y tomando como referencia al informe final emitido por equipo de auditores del Ministerio de Educación del Ecuador, menciona que la institución educativa alcanza el 72,84\% de cumplimiento de los estándares auditados, porcentaje que incluye la metodología de clase inversa, en la cual se acredita el indicador de calidad de color verde con su respectiva connotación de estándar "Satisfactorio".

Figura 1. Indicadores de calidad educativa. Fuente: Manual para la implementación y evaluación de los estándares de calidad educativa dimensión gestión pedgógica

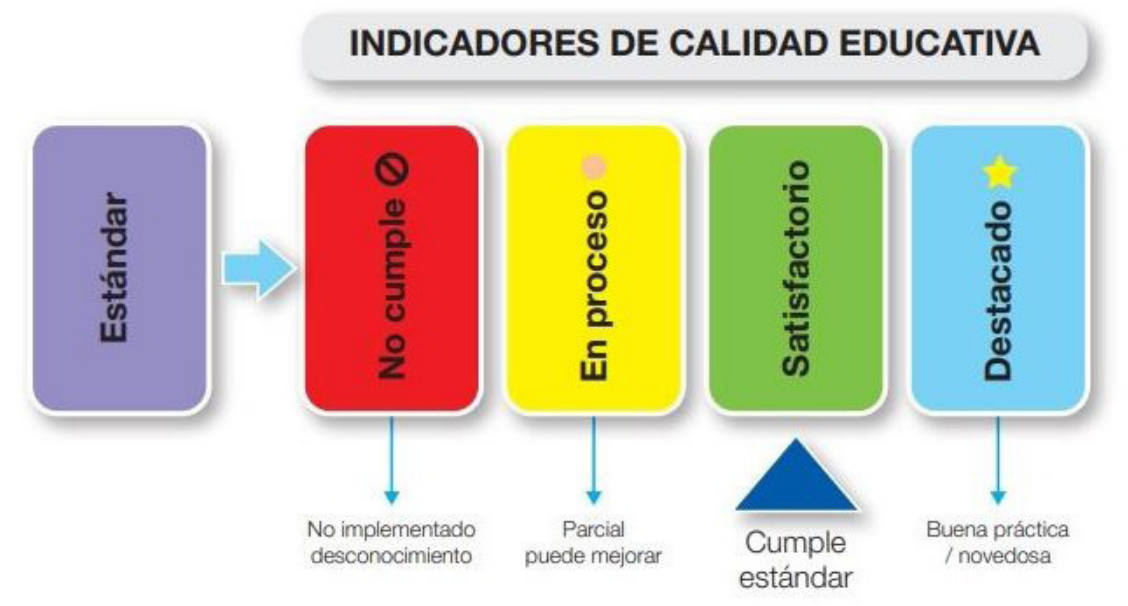

\section{MATERIAL Y MÉTODO}

\subsection{Herramientas TIC 2.0 en apoyo al trabajo colaborativo}

Considerando el mejorar los resultados de la auditoría realizada a la institución, se propone el uso de varias herramientas virtuales 2.0 en apoyo a la fase colaborativa de la metodología de clase inversa, promoviendo habilidades como: trabajo en equipo, resolución de problemas, comunicación y desarrollo de proyectos, las cuales se detallan a continuación: 


\subsection{Trabajo en equipo}

Según Guitert, Romeu y Pérez (2007), para analizar las competencias TIC que los estudiantes requieren para el trabajo en equipo, es necesario tomar en cuenta el escenario en el que se desenvuelven. Por ello en la institución previo al inicio del año lectivo, se considera la distribución física dentro del salón de clase, para lo cual en consenso entre docentes, se forma equipos de trabajo integrados por estudiantes con características académicas heterogéneas. Cada grupo cuenta con su respectivo líder, quien es seleccionado en función de habilidades como: capacidad de comunicarse, establecer metas, organización, buen desempeño académico, entre otras, de esta manera se garantiza un trabajo mancomunado y de calidad.

Ander-Egg (2001), afirma que principalmente se debe disponer equipos de trabajo que se complemente en la realización de actividades y tareas, en las que todos son responsables y den cumplimiento a un propósito común, esto se evidencia en los resultados al ejecutar una actividad.

A manera de distribuir equipos de trabajo y fortalecer la participación de sus integrantes es factible el uso de herramientas virtuales, que permitan un seguimiento por parte del docente, como se ejemplifica a continuación en la Figura 2:

Figura 2. Herramientas para el trabajo en equipo. Fuente: elaboración propia

\begin{tabular}{|c|c|c|c|c|c|c|}
\hline \multicolumn{7}{|c|}{ TRABAJO EN EQUIPO } \\
\hline \multirow[b]{2}{*}{ HERRAMienta } & \multicolumn{2}{|c|}{ UTILIDAD } & \multirow[b]{2}{*}{ ACTIVIDADES PARA EJECUTAR } & ACCESO & \multirow[b]{2}{*}{$\begin{array}{l}\text { LOGO DE LA } \\
\text { HERRAMIENTA }\end{array}$} & \multirow[b]{2}{*}{$\begin{array}{l}\text { FUENTE DE LA } \\
\text { IMAGEN }\end{array}$} \\
\hline & 量 & 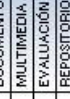 & & 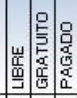 & & \\
\hline \multirow{2}{*}{ NOTION } & \multirow{2}{*}{$x$} & \multirow{2}{*}{$x \mid x$} & Sirve para la solución de problemas sociles & \multirow{2}{*}{$\mathrm{x}$} & & https://www.notio \\
\hline & & & Puede ser visto y editado por varias personas de forma simultánea & & & $\begin{array}{c}9 \mathrm{~b} 894077327940158 \\
0388 \mathrm{~d} 43 \mathrm{e} 8 \mathrm{c} 89 \mathrm{~d} 3 \mathrm{a}\end{array}$ \\
\hline \multirow{2}{*}{ Mindomo } & \multirow{2}{*}{$x$} & \multirow{2}{*}{$x$} & Es un software de creación de mapas mentales & \multirow{2}{*}{$\mathrm{x}$} & & \multirow[b]{2}{*}{$\begin{array}{l}\text { https://www.mind } \\
\text { omo.com/es/ }\end{array}$} \\
\hline & & & Los usuarios crean de manera colaborativa, pueden ver y compartir & & Mindomo & \\
\hline \multirow{2}{*}{$\begin{array}{l}\text { Google for } \\
\text { Education }\end{array}$} & \multirow{2}{*}{$x \mid x$} & \multirow{2}{*}{$x|x| x$} & Tienes funcionalidad similar a las suites de oficina tradicionales & \multirow{2}{*}{$\mathrm{x}$} & \multirow{2}{*}{$\begin{array}{l}\text { Google } \\
\text { for Education }\end{array}$} & \multirow{2}{*}{$\begin{array}{l}\text { https://tequilaintel } \\
\text { igente.com/2018/1 } \\
\text { 0/15/entrevista-a- } \\
\text { maria-teresa-arnal- }\end{array}$} \\
\hline & & & Contiene: Gmail , Hangouts, Google Calendar, Drive, Docs, Sheets & & & \\
\hline
\end{tabular}

\subsection{Resolución de problemas}

Uno de los factores que determinan el desarrollo del pensamiento crítico y la innovación es justamente la resolución de problemas en las que el estudiante debe involucrase de manera activa con el desarrollo de una sociedad más justa y equitativa para brindar soluciones a proble- 
mas de su entorno. En la institución objeto de estudio existe una amplia gama de opciones que los docentes utilizan para el cumplimiento del trabajo colaborativo pero en cierta forma se ha dado mayor importancia al contenido específico de cada asignatura más que al análisis de un problema social, se tiene acercamientos con programas de participación estudiantil impulsado por el Ministerio de Educación, que de acuerdo al interés del estudiante se integra en campos de acción como: convivencia, alimentación saludable, artístico cultural y ambiente; los cuales buscan generar un producto que aporte al desarrollo armónico de la comunidad educativa. Pero tampoco se puede desestimar los conocimientos previos de los estudiantes para la resolución de problemas como lo manifiestan Del Valle Coronel y Curotto (2008), al asegurar que muchas veces el fracaso de los estudiantes al tratar de dar solución a un problema se debe a la ausencia de habilidades y conocimientos.

El objetivo principal que constantemente tiene un equipo de trabajo es llegar a la solución de un determinado problema tanto de aspecto académico como de otro contexto, para lo cual, las herramientas tecnológicas pueden ser un aporte fundamental buscando la solución al conflicto que se presenta, siendo así, se sugiere herramientas online de fácil acceso (ver Figura 3).

Figura 3. Herramientas para resolución de problemas. Fuente: autores

\begin{tabular}{|c|c|c|c|c|c|c|c|}
\hline \multicolumn{8}{|c|}{ RESOLUCIÓN DE PROBLEMAS } \\
\hline \multirow[b]{2}{*}{ MERRAMIEMTA } & \multicolumn{3}{|c|}{ UTILIDAD } & \multirow[b]{2}{*}{ ACTIVIDADES PARA EJECUTAR } & ACCESO & \multirow[b]{2}{*}{\begin{tabular}{|} 
LOGO DE LA \\
HERRAMIENTA
\end{tabular}} & \multirow[b]{2}{*}{$\begin{array}{l}\text { FUENTE DE LA } \\
\text { IMAGEN }\end{array}$} \\
\hline & 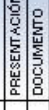 & 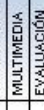 & 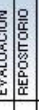 & & 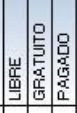 & & \\
\hline \multirow{2}{*}{$\begin{array}{l}\text { Pollevery } \\
\text { where }\end{array}$} & \multirow{2}{*}{$x}$, & \multirow{2}{*}{$x \mid x$} & \multirow{2}{*}{$x \mid x$} & Software en línea en tiempo real para la elaboración cuestionarios & \multirow{2}{*}{$x \mid x$} & \multirow{2}{*}{ (1) Poll Exerymber } & \multirow{2}{*}{$\frac{\text { https://www.pollev }}{\text { erywhere.com/ }}$} \\
\hline & & & & Elabora encuestas en línea sin depender de un número de usuarios & & & \\
\hline \multirow{2}{*}{ Screencast } & \multirow{2}{*}{$x \mid x$} & \multirow{2}{*}{$x \mid x$} & \multirow{2}{*}{$\times$} & Facilita grabación digital en una pantalla con audio y video. & \multirow{2}{*}{$x$} & \multirow{2}{*}{$\begin{array}{c}\text { Screencast } \\
\text { Matic }\end{array}$} & \multirow{2}{*}{$\begin{array}{c}\text { https://screencast- } \\
\text { o- } \\
\text { matic.com/screen- } \\
\text { recorder }\end{array}$} \\
\hline & & & & Permite una edición de sofisticadas de los archivos grabados. & & & \\
\hline \multirow{2}{*}{ Goconar } & \multirow{2}{*}{$x|x| \times$} & \multirow{2}{*}{\multicolumn{2}{|c|}{$x \mid x$}} & Es un apoyo al estudiante por medio de la representación de diagramas & \multirow{2}{*}{$x \mid x$} & \multirow{2}{*}{ goconar } & \multirow{2}{*}{$\begin{array}{c}\text { https://www.gocon } \\
\text { qr.com/es/ }\end{array}$} \\
\hline & & & & Crea diversos organizadores gráficos y diagramas & & & \\
\hline
\end{tabular}

\subsection{Comunicación}

Los estudiantes deberán desarrollar habilidades de comunicación, pero esta no debe simplemente enfrascarse en la transmisión de algún tipo de información sino más bien como la conjugación de significados que den relevancia, muchas veces se cae en el error de convertirla en un instrumento sesgado e individual del docente, la comunicación debe estar abierta a los intereses de todos los involucrados de una manera multidisciplinaria y no debe ser individualizada por una o varias personas que crean tener una verdad irrefutable, promoviendo el 
desarrollo progresivo de la comunicación dentro del salón de clase como lo afirma Báez (2000), utilizando un lenguaje común que otorgue un sentido de pertenencia, logrando que exista mayor comunicación y por ende mayor cohesión grupal. Además se debe analizar los medios de manera que se efectivicen, se concuerda con Coll, Mauri y Onrubia (2008), quienes mencionan que el aprovechamiento de las TIC depende en gran medida de la disposición de los equipos necesarios con los que se cuente el establecimiento, lamentablemente las instituciones en el país de sostenimiento fiscal aún no disponen de una capacidad en tecnología, sobre todo en el aula de clase, sin embargo en la institución se realizan esfuerzos para cubrir esa brecha.

La manera más efectiva de lograr un trabajo en colaboración con otras personas es tener una comunicación de calidad, misma que debe apoyarse en la tecnología, rompiendo el esquema de tiempo y distancia, por tanto, se recomienda el uso de las siguientes herramientas virtuales.

Figura 4. Herramientas para la comunicación. Fuente: elaboración propia

\begin{tabular}{|c|c|c|c|c|c|c|c|}
\hline \multicolumn{8}{|c|}{ COMUNICACIÓN } \\
\hline \multirow[b]{2}{*}{ MERRAMIEMTA } & \multicolumn{3}{|c|}{ UTILIDAD } & \multirow[b]{2}{*}{ ACTIVIDADES PARA EJECUTAR } & ACCESO & \multirow[b]{2}{*}{$\begin{array}{l}\text { LOGO DE LA } \\
\text { HERRAMIENTA }\end{array}$} & \multirow[b]{2}{*}{$\begin{array}{l}\text { FUENTE DE LA } \\
\text { IMAGEN }\end{array}$} \\
\hline & 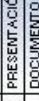 & 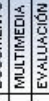 & 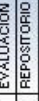 & & 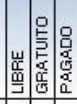 & & \\
\hline \multirow{2}{*}{ Oovoo } & \multirow{2}{*}{$\mathrm{x}$} & \multirow{2}{*}{$x \mid x$} & \multirow{2}{*}{$x \mid x$} & Es factible llamadas en grupo de hasta 12 participantes y grabarlas & \multirow{2}{*}{$\mathrm{x}$} & \multirow{2}{*}{ oolbo } & \multirow[b]{2}{*}{$\begin{array}{c}\text { https://www.oovo } \\
\text { o. com/ }\end{array}$} \\
\hline & & & & Compartir la pantalla y enviar videomensajes a los contactos & & & \\
\hline \multirow{2}{*}{ Zoom } & \multirow{2}{*}{$x \mid x$} & \multirow{2}{*}{\multicolumn{2}{|c|}{$x|x|>$}} & Videoconferencias a grandes grupos y una excelente calidad en imagen & \multirow{2}{*}{$\mathrm{x}$} & \multirow{2}{*}{ zoom } & \multirow{2}{*}{ https://zoom.us/ } \\
\hline & & & & Puede ser usado como chat en linea de manera sincrónica & & & \\
\hline \multirow{2}{*}{ Slack } & \multirow{2}{*}{$x|x|$} & \multirow{2}{*}{\multicolumn{2}{|c|}{$x|x| x$}} & Facilita la comunicación con varios grupos de trabajo públicos y privados & \multirow{2}{*}{$x$} & \multirow{2}{*}{ "े. slack } & \multirow{2}{*}{$\begin{array}{c}\text { https://slack.com/i } \\
\text { ntl/es-ec/ }\end{array}$} \\
\hline & & & & Puede compratir archivos en deferentes formatos & & & \\
\hline
\end{tabular}

\subsection{Desarrollo de proyectos}

En la institución, al finalizar cada unidad didáctica, se elabora un proyecto llamado "Proyecto de desempeño" en el que se plasma y pone en evidencia las habilidades adquiridas o mejoradas durante un determinado período, presentando un producto útil para la sociedad, como lo mencionan Badia y García (2006), el desarrollo de proyectos no solo es una simple recopilación de datos en un documento, en su lugar debe demostrar el desarrollo cognitivo alcanzado por los estudiantes. Es importante destacar el aprendizaje basado en proyectos ABP como referencia para su óptima aplicación integrándolos a las TIC como apoyo sustancial, así lo afirman Martí, Heydrich, Rojas y Hernández (2010), al exponer que las TIC ya nos son herramientas individuales para los estudiantes, sino que ahora además son un soporte para el aprendizaje colaborativo y el complemento en la adquisición de conocimientos conjuntos. 
Elaborar proyectos de manera digital permite un control en tiempo y espacio por todos sus integrantes, que a su vez garantizarán el éxito de las actividades planteadas, por tanto, se propone las siguientes herramientas virtuales (Figura 5).

Figura 5. Herramientas para desarrollo de proyectos. Fuente: elaboración propia

\begin{tabular}{|c|c|c|c|c|c|c|}
\hline \multicolumn{7}{|c|}{ DESARROLLO DE PROYECTOS } \\
\hline \multirow[b]{2}{*}{ MERRAMIERTA } & \multicolumn{2}{|c|}{ UTILIDAD } & \multirow[b]{2}{*}{ ACTIVIDADES PARA EJECUTAR } & ACCESO & \multirow[b]{2}{*}{$\begin{array}{l}\text { LOGO DE LA } \\
\text { HERRAMIENTA }\end{array}$} & \multirow[b]{2}{*}{$\begin{array}{l}\text { FUENTE DE LA } \\
\text { IMAGEN }\end{array}$} \\
\hline & 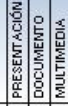 & 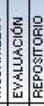 & & 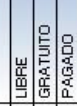 & & \\
\hline \multirow{2}{*}{ Trello } & $x$ & \multirow{2}{*}{$\times \times$} & Favorece la planificación y elaboración de proyectos sobre temas varios & \multirow{2}{*}{$x$} & all. & \multirow{2}{*}{ https://trello.com/ } \\
\hline & & & Asigna tareas especificas a cada miembro del equipo & & & \\
\hline \multirow{2}{*}{ google sites } & \multirow{2}{*}{$x \mid x$} & \multirow{2}{*}{$x \mid x$} & Fomenta la creación de páginas web sencillas & \multirow{2}{*}{$x$} & \multirow{2}{*}{ D Google Stes } & \multirow{2}{*}{$\begin{array}{l}\text { https://sites.google } \\
\text {.com/new?hl=ES }\end{array}$} \\
\hline & & & Todos los colaboradores pueden modificar la página según las necesidades & & & \\
\hline \multirow{2}{*}{ Toggl } & \multirow{2}{*}{$x|x| x$} & \multirow{2}{*}{$x \mid x$} & Es una línea del tiempo de proporciona el desarrollo de un proyecto & \multirow{2}{*}{$x \mid x$} & \multirow{2}{*}{ (C) toggl } & \multirow{2}{*}{ https://toggl.com/ } \\
\hline & & & Los proyetos pueden ser visualizados publicamente & & & \\
\hline
\end{tabular}

\subsection{Método}

En base a la revisión de la literatura, se ha considerado indispensable para la presente investigación, el establecer estas fases que integran los siguientes aspectos:

Figura 6. Fases de la revisión de la Literatura. Fuente: elaboración propia

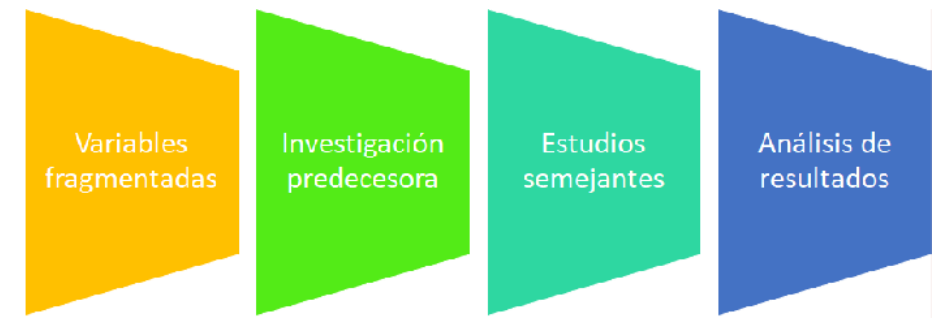

\section{A. Variables fragmentadas}

Consiste en la recopilación de investigaciones relevantes a nivel mundial relacionadas con clase inversa, trabajo colaborativo y las TIC.

\section{B. Investigación predecesora}

Se sustenta en los resultados en la investigación predecesora misma que plasma de forma general las fases de clase inversa y la presente se enfatiza en aspectos relevantes como trabajo colaborativo. 


\section{Estudios semejantes}

Las investigaciones que dan aporte fundamental a la presente tienen impacto en el contexto que se desarrollaron y a la vez brindan un aporte sustancial al proceso de aprendizaje.

\section{Análisis de resultados}

Es pertinente que el trabajo colaborativo se encuentre estrechamente relacionado a la actualidad apoyándose en la tecnología.

Los resultados de las investigaciones observadas demuestran la efectividad de las TIC en apoyo al trabajo colaborativo.

Los trabajos investigativos encontrados en relación al presente estudio fueron tomados en cuenta por su relevancia y la actualidad de los mismos, logrando determinar que existen escasas investigaciones sobre el tema presentado, siendo un total de 28 publicaciones relacionadas de manera directa, como se evidencia en la Tabla 1.

Tabla 1. Resultados tipos de publicaciones. Fuente: elaboración propia

\begin{tabular}{cc}
\hline TIPO DE PUBLICACIÓN & TOTAL \\
\hline Revistas & 5 \\
\hline Conferencias & 8 \\
\hline Tesis & 12 \\
\hline Libros / Capítulos de libros & 3 \\
\hline TOTAL & 28 \\
\hline
\end{tabular}

De esta manera, se fundamenta en una investigación aplicada apoyada en un enfoque cuantitativo, promoviendo como variable la indagación a las TIC en el apoyo al trabajo colaborativo en aula invertida, teniendo como propósito identificar los resultados que esta puede suscitar en los estudiantes dentro de un salón de clase; cabe indicar que el uso de las TIC no tiene que ser una elección al azar, por consiguiente deben ser planificadas con antelación a la clase por parte del docente y plasmadas en la planificación por destreza.

\section{RESULTADOS}

Basado en las habilidades del siglo XXI que se pretenden desarrollar en la etapa de trabajo colaborativo de clase inversa, se propuso la elaboración de una planificación por destreza incorporando las TIC (Ver Anexo 1), misma que a la vez debe cumplir con los contenidos e 
indicadores de logro que se requieren obtener en los estudiantes, la cual se aplicó como referencia en la asignatura de Lengua y Literatura en estudiantes de tercero curso de Bachillerato General Unificado (BGU).

Es importante destacar las experiencias de aprendizaje que se maneja en la planificación por destreza, donde se puede evidenciar como prevalece el uso de las TIC tanto en el objetivo, actividad previa y actividad colaborativa, teniendo en cuenta que lo más importante en estas etapas es la selección eficaz de las diversas herramientas tecnológicas, que el profesor elige en correspondencia a las estrategias y técnicas pedagógicas, para lograr un aprendizaje significativo.

\subsection{Análisis comparativo de resultados}

En base al producto de una planificación que incorpora el uso de las TIC dentro de un salón de clase, se realizó un análisis de promedios de las notas de rendimiento académico, entre parciales en el quimestre 2 del año lectivo 2018-2019, comparando con los parciales 4 y 5 , siendo estos planificados de forma regular sin el uso de herramientas tecnológicas y el parcial 6 como objeto de estudio según la planificación planteada con el uso de TIC, obteniendo los siguientes resultados.

Figura 7. Planificación según fases de clases inversa incorporando las TIC. Fuente: elaboración propia

\begin{tabular}{|c|c|c|c|c|}
\hline \multicolumn{2}{|c|}{ SEMANA: } & 1 & DESDE- HASTA: & 9 al 13 de septiembre 2019 \\
\hline \multicolumn{5}{|c|}{ MEDIACIÓN DE EXPERIENCIAS DE APRENDIZAJE } \\
\hline Objetivo: & $\begin{array}{l}\text { Ident } \\
\text { la cor } \\
\text { de es }\end{array}$ & uatc & $\begin{array}{l}\text { cas correspondiente } \\
\text { diante la investigaci } \\
\text { ron en la sociedad. }\end{array}$ & $\begin{array}{l}\text { rrupo de la Sierra que pertenecieron a } \\
\text { stentada con el apoyo de las TIC, para }\end{array}$ \\
\hline Actividad previa: & \multicolumn{4}{|c|}{$\begin{array}{l}\text { Docente: } \\
\text { 1. Realizar la distribución de equipos de estudiantes de } 4 \text { personas y distribuir las biografías de los } \\
\text { escritores del grupo de la Sierra. } \\
\text { Estudiantes: } \\
\text { 1. Investigar en YouTube la biografía de Jorge Icaza, Fernando Chávez, Humberto Salvador, Enrique } \\
\text { 2. Conćn, Jorge Fernández, Humberto Mata, Alfonso Cuesta y Ángel Felicísimo Rojas. } \\
\text { adecuadamente sus responsabilidades (Responsabilidad del líder de equipo). }\end{array}$} \\
\hline Actividad individual: & \multicolumn{4}{|c|}{ Responda la pregunta ¿A qué Corriente Literaria ecuatoriana pertenece este grupo de escritores? } \\
\hline Actividad colaborativa: & \multicolumn{4}{|c|}{$\begin{array}{l}\text { Analizar: Los aspectos más relevantes de la biografía del escritor. } \\
\text { Discutir: La (s) obras que representan la esencia del escritor. } \\
\text { Acordar: La elaboración de un diagrama u organizador gráfico en (borrador). } \\
\text { Usar la herramienta tecnológica: Creately para realizar la presentación. }\end{array}$} \\
\hline Actividad de clase: & \multicolumn{4}{|c|}{$\begin{array}{l}\text { Estudiantes: Exponer la biografía de escritor (asignado previamente). } \\
\text { Docente: Al final de cada exposición profundizar los contenidos. }\end{array}$} \\
\hline Actividad de seguimiento: & \multicolumn{4}{|c|}{$\begin{array}{l}\text { Estudiante: Realizar un vídeo sobre la biografía del escritor (asignado previamente) en la herramienta Explee. } \\
\text { El link debe compartirlo en la plataforma http://schoology.com/ }\end{array}$} \\
\hline
\end{tabular}


Figura 8. Resultados de planificación por destreza con TIC. Fuente: elaboración propia

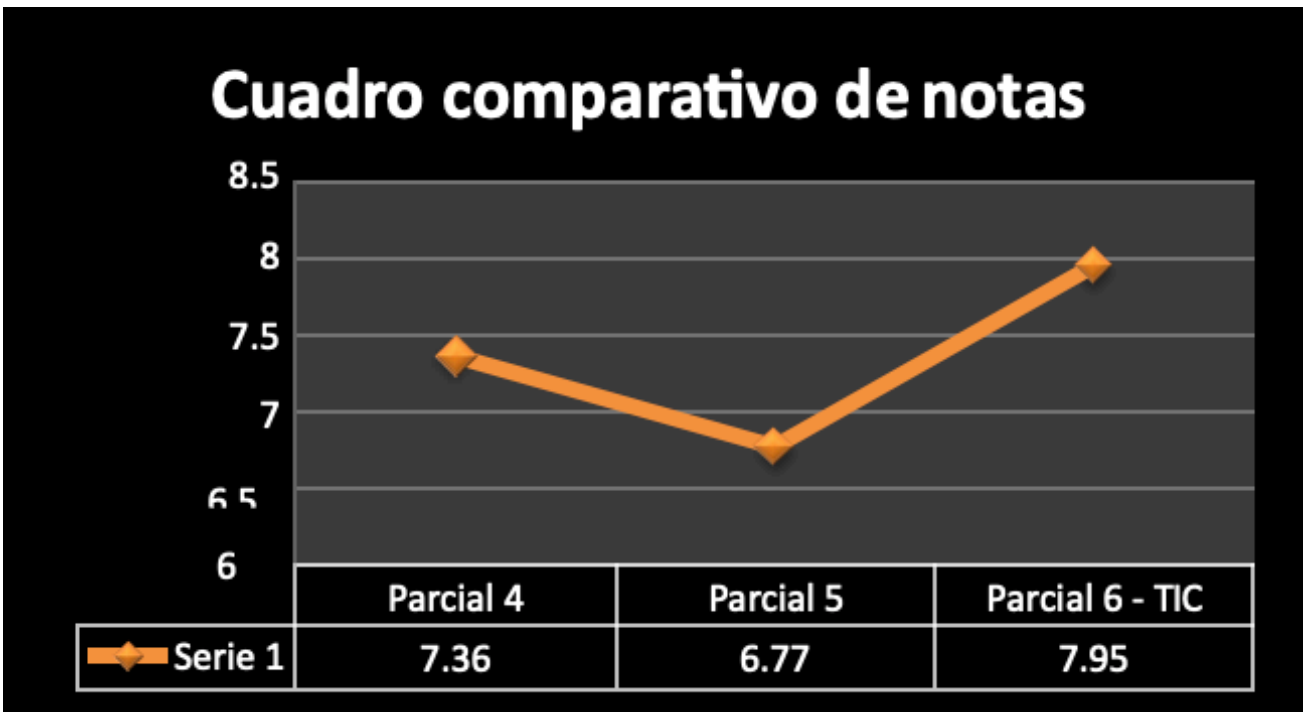

Con base a los resultados obtenidos en el rendimiento académico, se registra un aumento del 5,9\% entre el parcial 4 al 6; a la vez existe un incremento del 11,8\% entre el parcial 5 al 6, lo que podría fortalecer la premisa que entre varios factores las TIC son una fuente de motivación para los estudiantes y así alcanzar un aprendizaje significativo mejorando su rendimiento académico.

\section{DISCUSIÓN}

Las actividades colaborativas con apoyo de las TIC en un salón de clase, son relativamente nuevas dentro del contexto educativo, donde muchos docentes se han mostrado reacios al cambio de paradigma y se mantienen en modelos tradicionales. Sin embargo, la tendencia se distorsiona favorablemente en instituciones que han optado por innovar como lo aseguran García, Basillota y López (2014), al manifestar que el aprendizaje colaborativo genera ventajas en el desarrollo de competencias transversales, posibilitando el desarrollo de habilidades, las cuales con el uso de TIC enlazan perfectamente la motivación y la autoestima.

Por otro lado, al realizar trabajos colaborativos virtuales según Fernández et al. (2020) los estudiantes tienen la oportunidad de construir un aprendizaje social, sistematizando e intercambiando ideas que les permitan realizar determinadas actividades, lo cual se refleja en un producto íntegro. 
Del mismo modo es menester conocer las opiniones de los estudiantes que utilizan herramientas tecnológicas durante el trabajo colaborativo como lo aseguran Soto y Torres (2016), al evidenciar que los estudiantes aceptan y valoran las actividades colaborativas en vista de la relación de la teoría con la práctica colectiva. Por consiguiente, también los docentes siendo guías del proceso de enseñanza- aprendizaje saben lo fundamental de llevar la teoría a la práctica, siendo esta la encargada de forjar un aprendizaje significativo en los estudiantes, así como lo hace notar Aguirre, González y González (2012), para obtener un aprendizaje significativo el estudiante puede apoyarse en diversidad de medios que faciliten una agilidad en la búsqueda de información, por lo que, la Web 2.0 puede favorecer en esta necesidad individual, promoviendo de manera futura que el estudiante exponga sus conocimientos en un grupo de trabajo expresando los saberes ya adquiridos y a la vez todos los integrantes del mencionado grupo compartan lo que han logrado, por lo tanto, consiguiendo apropiarse de un aprendizaje significativo en conjunto.

Considerando que el salón de clase es un espacio físico el cual debe afianzar una gran cantidad de conocimientos se estima pertinente el relacionar saberes empíricos y recientes. Según Ausubel (1983), "el aprendizaje del alumno depende de la estructura cognitiva previa que se relaciona con la nueva información, debe entenderse por estructura cognitiva, al conjunto de conceptos, ideas que un individuo posee en un determinado campo del conocimiento" (p. 1). En ese sentido, la concreción de un aprendizaje procede de una investigación profunda de hechos y acontecimientos procurando un análisis minucioso en búsqueda de obtener una información autentica, con ello, establecer un discernimiento para obtener una compresión que perdure en tiempo y espacio.

El proceso de aprendizaje a lo largo del tiempo se ha considerado para los estudiantes en una meta a cumplir; consiente el docente del reto académico que debe ayudar a culminar tiene la misión de considerar factores que favorezcan la atención del estudiante en su diario aprender. Según García y Doménech (1997):

Habitualmente la motivación académica ha sido tratada desde la perspectiva de la persona; es decir como una variable personal y haciendo referencia a los componentes que la integran (autoconcepto, atribuciones causales y metas de aprendizaje, emociones, etc.), sin prestar demasiada atención a los factores contextuales y en el modo en que éstos pueden influir en la motivación. Sin embargo, es importante señalar que estas variables personales que hemos tratado están estrechamente condicionadas por el ambiente en el cual el niño/a desarrolla su actividad. (p. 18) 
Tal como lo menciona Escobar (2016), el uso de recursos digitales en varias asignaturas dinamiza e impulsa el desarrollo de competencias esenciales y comunicacionales en los estudiantes favoreciendo además un razonamiento lógico, pensamiento crítico y resolución de conflictos cotidianos. En consecuencia, las investigaciones enfocadas en el uso de TIC en educación apuntan a la necesidad de generar ambientes motivadores dentro del salón de clase con el uso de material digital.

\section{CONCLUSIONES}

Se ha demostrado que el uso efectivo de las TIC como apoyo sustancial y motivante dentro del proceso de enseñanza-aprendizaje genera resultados favorables que van a la par con las nuevas tendencias metodológicas, y sobre las cuales las instituciones educativas deberían poner énfasis para contribuir al desarrollo de un aprendizaje significativo, con el rediseño de sus estructuras pedagógicas y curriculares que garanticen una apropiada construcción del conocimiento.

La integración congruente de las fases de clase inversa crea una base sobre la cual docentes y estudiantes trabajan con un mayor compromiso y dedicación, con miras a un nuevo enfoque que rompe el esquema tradicional de una clase orientándola al fomento de actividades dinámicas y participativas centradas en el estudiante y que conllevan al desarrollo o la repotenciación de habilidades necesarias para que el estudiante se enfrente a los desafíos del contexto actual.

Las actividades colaborativas dentro del salón de clase impulsan al desarrollo de habilidades como el trabajo en equipo, la resolución de problemas, la comunicación y el desarrollo de proyectos que al enlazarlos con el apoyo de las TIC generan además un incremento en el rendimiento académico de los estudiantes tal como se lo ha demostrado en el presente estudio al exponer un plan de clase utilizando la metodología de clase inversa con el apoyo de TIC.

Los resultados obtenidos en la presente investigación podrían servir como base para futuros trabajos en los que se destaque la efectividad del trabajo colaborativo en otros niveles de educación, por ejemplo, en educación general básica, ya que se ha puesto de manifiesto que la realización de actividades colaborativas provoca un vínculo de cooperación entre los estudiantes permitiéndoles desarrollar oportunamente una comunicación asertiva. 
ANEXO 1

UNIDAD EDUCATIVA SAN FRANCISCO DE QUITO

"Educar con calidad, es educar con amor y excelencia" 2019-2020

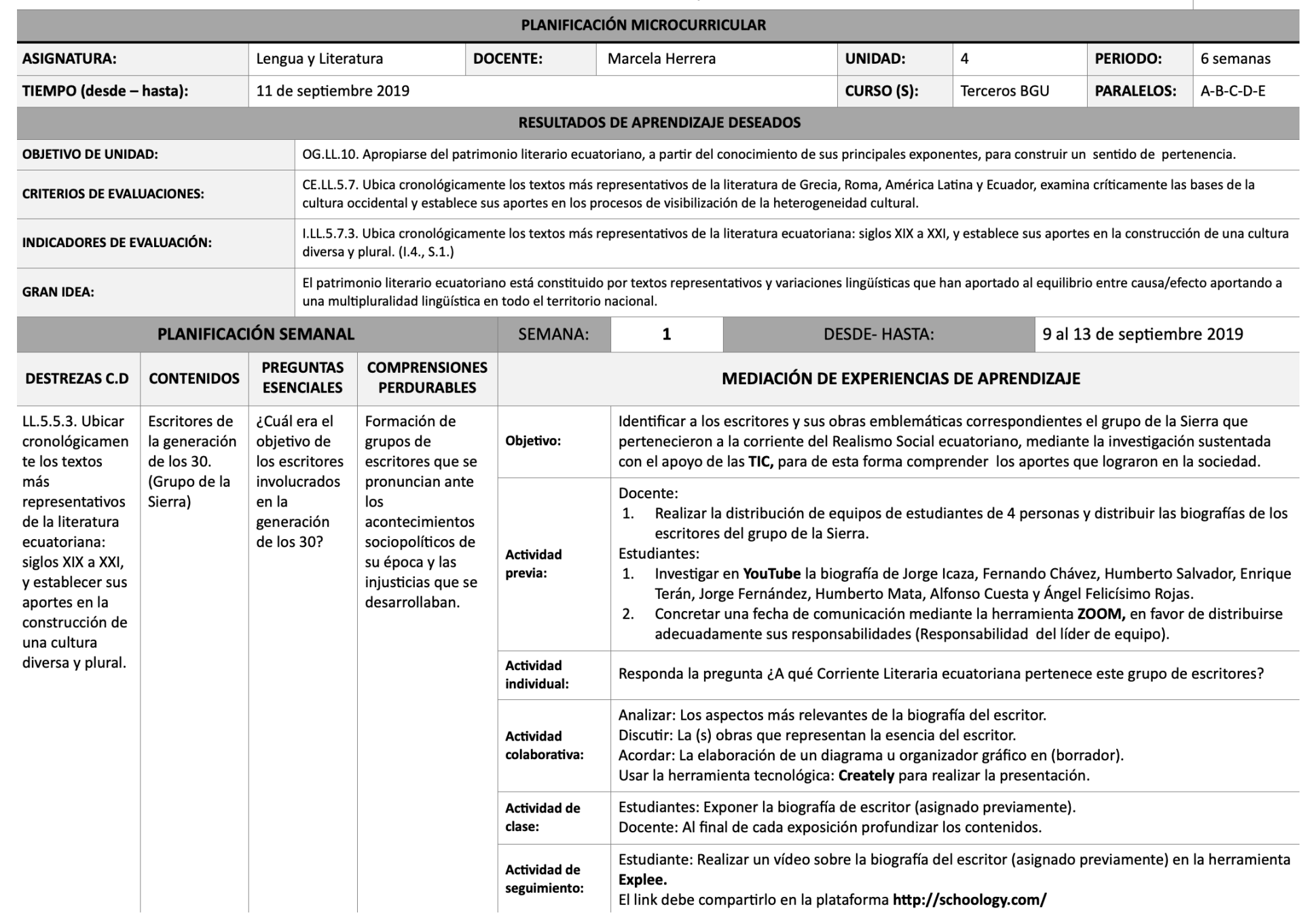

\section{REFERENCIAS BIBLIOGRÁFICAS}

Aguirre, E. I. R., González, N. L. M., \& González, R. M. G. (2012). Aprendizaje colaborativo en ambientes virtuales y sus bases socioconstructivistas como vía para el aprendizaje significativo. Apertura, $4(2), 32-41$.

Ander-Egg. (2001). El trabajo en equipo. Editorial Progreso.

Ausubel, D. (1983). Teoría del aprendizaje significativo. Fascículos de CEIF, 1(1-10). 
Badia, A., \& García, C. (2006). Incorporación de las TIC en la enseñanza y el aprendizaje basados en la elaboración colaborativa de proyectos.

Báez, C. J. (2000). La comunicación efectiva. Intec.

Coll, C., Mauri, T., \& Onrubia, J. (2008). La utilización de las tecnologías de la información y la comunicación en la educación: Del diseño tecno-pedagógico a las prácticas de uso. Psicología de la educación virtual, 74-103.

Calzadilla, M. E. (2002). Aprendizaje colaborativo y tecnologías de la información y la comunicación. Revista Iberoamericana de educación, 29(1), 1-10.

Del Valle Coronel, M., \& Curotto, M. M. (2008). La resolución de problemas como estrategia de enseñanza y aprendizaje. Revista electrónica de enseñanza de las ciencias, 7(2), 464.

Escobar Zapata, F. A. (2016). El uso de las TIC como herramienta pedagógica para la motivación de los docentes en el proceso de aprendizaje y enseñanza en la asignatura de inglés.

Férez, P. E. G. (2005). Un acercamiento al trabajo colaborativo. Revista iberoamericana de educación, 36(7), 1-14.

Fernández, A. C., Valderrey, V. M., Lázaro, I. G., Gil-Mediavilla, M., \& Gallardo-López, J. A. (2020). El trabajo colaborativo online como herramienta didáctica en Espacios de Enseñanza Superior (EEES). Percepciones de los estudiantes de los Grados en Educación Infantil y Primaria. Revista d’Innovació Docent Universitària, (12), 82-94.

Ferreiro, Ramón (2012). La pieza clave del rompecabezas del desarrollo de la creatividad: la escuela. REICE. Revista Iberoamericana sobre Calidad, Eficacia y Cambio en Educación, 10(2),6-22.https:// www.redalyc.org/articulo.oa?id=551/55124596002

García Bacete, F. J., \& Doménech Betoret, F. (1997). Motivación, aprendizaje y rendimiento escolar.

García, A., Basilotta, V., \& López, C. (2014). Las TIC en el aprendizaje colaborativo en el aula de Primaria y Secundaria. Comunicar, 21(42), 65-74.

Guitert, M., Romeu, T., \& Pérez-Mateo, M. (2007). Competencias TIC y trabajo en equipo en entornos virtuales. RUSC. Universities and Knowledge Society Journal, 4(1).

Herrera Mueses, M. L., Perugachi Mediavilla, J. I., \& Baldeón Egas, P. F. (2019). Las TIC en el desarrollo de clase inversa: experiencia Unidad Educativa Fiscal San Francisco de Quito. Revista Conrado, 15(70), 248-257. https://conrado.ucf.edu.cu/index.php/conrado/article/view/1133

IBEC. (2019). Habilidades del siglo 21. p. 2 
Martí, J. A., Heydrich, M., Rojas, M., \& Hernández, A. (2010). Aprendizaje basado en proyectos. Revista Universidad EAFIT, 46(158).

Ministerio de Educación del Ecuador. (2010). Actualización y Fortalecimiento Curricular de la Educación General Básica. p.16.

Ministerio de Educación de Ecuador (2017). Manual para la implementación y evaluación de los estandares de calidad educativa. p.17

Soto Ortiz, J. L., \& Torres Gastelú, C. A. (2016). La percepción del trabajo colaborativo mediante el soporte didáctico de herramientas digitales. Apertura (Guadalajara, Jal.), 8(1), 0-0. 